AperTO - Archivio Istituzionale Open Access dell'Università di Torino

Increased serum ferritin levels predict long-term mortality in patients with NAFLD

This is a pre print version of the following article:

Original Citation:

Availability:

This version is available http://hdl.handle.net/2318/1779745

since 2021-03-12T13:11:38Z

Terms of use:

Open Access

Anyone can freely access the full text of works made available as "Open Access". Works made available under a Creative Commons license can be used according to the terms and conditions of said license. Use of all other works requires consent of the right holder (author or publisher) if not exempted from copyright protection by the applicable law. 


\section{Increased serum ferritin levels predict long-term mortality in patients with NAFLD}

A. Armandi 1 , R. Younes 2 , G.P. Caviglia 1, S. Petta 3 , L. Miele 4 , 5 , C. Rosso 1 , G. Pennisi 3 , P. Francione 6 , A. Liguori 5 , O. Govaere 7 , A.L. Fracanzani 6 , M. Eslam 8, L. Valenti 6 , J. George 8, Q.M. Anstee 7 , E. Bugianesi 1

1 Department of Medical Sciences, Division of Gastroenterology and Hepatology, A.O. Città della Salute e della Scienza di Torino, University of Turin, Turin, Italy

2 Boehringer Ingelheim International, GmbH, Ingelheim, Germany

3 Sezione di Gastroenterologia, Dipartimento Biomedico di Medicina Interna e Specialistica, Universita ' di Palermo, Palermo, Italy

4 Area Medicina Interna, Gastroenterologia e Oncologia Medica, Fondazione Policlinico A. Gemelli IRCCS, Rome, Italy

5 Universita' Cattolica del Sacro Cuore, Rome, Italy

6 Unit of Medicine and Metabolic Disease, Department of Pathophysiology and Transplantation, Ca' Granda IRCCS Foundation, Policlinico Hospital, University of Milan, Milan, Italy

7 The Newcastle Liver Research Group, Translational and Clinical Research Institute, Faculty of Medical Sciences, Newcastle University, Newcastle upon Tyne, United Kingdom

8 Storr Liver Centre, Westmead Institute for Medical Research, Westmead Hospital and University of Sydney, Westmead, NSW, Australia

Background and Aim: Hyperferritinemia is a common feature in patients with non-alcoholic fatty liver disease (NAFLD) and correlates with the severity of liver fibrosis. Our aim was to assess the impact of ferritin on long-term outcomes and survival in a large cohort of NAFLD patients.

Methods: We included 1247 patients with biopsy-proved NAFLD in tertiary centers in Italy (Turin, Milan, Rome, Palermo), Australia (Sydney) and UK (Newcastle). Clinical and biochemical data were collected at the time of liver biopsy. Ferritin levels $\leq 300 \mu \mathrm{g} / \mathrm{L}$ for men and $\leq 200 \mu \mathrm{g} / \mathrm{L}$ for women were considered as upper limit of normal (ULN). Clinical outcomes, including liver-related events (ascites, encephalopathy, variceal bleeding) and survival, were collected after a median follow-up of 90 months.

Results: The median age of the study cohort was 48 [IQR 38-57] years and 814 (65.3\%) patients were males. The prevalence of obesity and type 2 diabetes was $45.0 \%$ and $28.1 \%$. Overall, hyperferritinemia was found in $373(29.9 \%)$ patients; severe fibrosis $(\mathrm{F} \geq 3)$ was found at liver biopsy in $272(21.8 \%)$ patients while NASH was diagnosed in $756(60.6 \%)$ of cases. Serum ferritin $>2 \times U L N$ resulted significantly associated with $\mathrm{F} \geq 3(\mathrm{OR}=2.10,95 \% \mathrm{CI} 1.40-3.14, \mathrm{p}<0.001)$. After a median follow-up of 90 months, 24 patients (2.3\%) died and 57 (4.8\%) developed liverrelated events. At univariate analysis, the incidence of liver-related events and mortality varied significantly according to serum ferritin values $>2 x U L N($ Log-rank test: $p=0.004$ and $p=$ 0.001 , respectively). However, at multivariate Cox regression analysis adjusted for age, body mass index, diabetes and fibrosis, ferritin levels $>2 x U L N$ independently predicted mortality $(\mathrm{HR}=3.04$, $95 \%$ CI 1.16-7.93, $\mathrm{p}=0.023)$ but not liver-related events $(\mathrm{HR}=1.67,95 \% \mathrm{CI} 0.90-3.11, \mathrm{p}=0.105)$.

Conclusions: Ferritin levels higher than $2 \mathrm{xULN}$ are associated to severe liver fibrosis in NAFLD patients and are able to predict longterm mortality.

This work has received support from the EU/EFPIA Innovative Medicines Initiative 2 Joint Undertaking (LITMUS grant no. 777377). 